PROCEEDINGS OF THE

AMERICAN MATHEMATICAL SOCIETY

Volume 130, Number 1, Pages 73-79

S 0002-9939(01)06156-1

Article electronically published on May 3, 2001

\title{
ON THE $L^{p}$ BOUNDEDNESS OF THE NON-CENTERED GAUSSIAN HARDY-LITTLEWOOD MAXIMAL FUNCTION
}

\author{
LILIANA FORZANI, ROBERTO SCOTTO, \\ PETER SJÖGREN, AND WILFREDO URBINA
}

(Communicated by David Preiss)

\begin{abstract}
The purpose of this paper is to prove the $L^{p}\left(\mathcal{R}^{n}, d \gamma\right)$ boundedness, for $p>1$, of the non-centered Hardy-Littlewood maximal operator associated with the Gaussian measure $d \gamma=e^{-|x|^{2}} d x$.
\end{abstract}

Let $d \gamma=e^{-|x|^{2}} d x$ be a Gaussian measure in Euclidean space $\mathcal{R}^{n}$. We consider the non-centered maximal function defined by

$$
\mathcal{M} f(x)=\sup _{x \in B} \frac{1}{\gamma(B)} \int_{B}|f| d \gamma,
$$

where the supremum is taken over all balls $B$ in $\mathcal{R}^{n}$ containing $x$. P. Sjögren [2] proved that $\mathcal{M}$ is not of weak type $(1,1)$ with respect to $d \gamma$ for $n>1$. A more general result was obtained by A. Vargas [4, who characterized those radial and strictly positive measures for which the corresponding maximal operator is of weak type $(1,1)$. However, these papers leave open the question of the $L^{p}(d \gamma)$ boundedness of $\mathcal{M}$ for $p>1$ and $n>1$.

The main result in this paper is

Theorem 1. $\mathcal{M}$ is a bounded operator on $L^{p}(d \gamma)$ for $p>1$, that is, there exists a constant $C=C(n, p)$ such that for $f \in L^{p}(d \gamma)$,

$$
\|\mathcal{M} f\|_{L^{p}(d \gamma)} \leq C\|f\|_{L^{p}(d \gamma)}
$$

In a forthcoming paper [3], P. Sjögren and F. Soria prove estimates for the maximal operator associated with a more general radial measure with decreasing density.

We denote $S_{r}^{n-1}=\left\{x \in \mathcal{R}^{n}:|x|=r\right\}$ and $S^{n-1}=S_{1}^{n-1}$, and write $d \sigma$ for the area measure on $S^{n-1}$. The spherical maximal function

$$
\mathcal{M}^{e} f(h)=\sup _{R>0} \frac{1}{\sigma\left(\left|z^{\prime}-h\right| \leq R\right)} \int_{\left|z^{\prime}-h\right| \leq R}\left|f\left(z^{\prime}\right)\right| d \sigma\left(z^{\prime}\right), \quad h \in S^{n-1},
$$

is bounded on $L^{p}(d \sigma)$. We extend $\mathcal{M}^{e}$ to functions defined in $\mathcal{R}^{n}$ by using polar coordinates $x=\rho x^{\prime}$ with $x^{\prime} \in S^{n-1}$ and applying $\mathcal{M}^{e}$ in the $x^{\prime}$ variable. Then $\mathcal{M}^{e}$ is bounded on $L^{p}(d \gamma)$.

Received by the editors May 15, 2000.

1991 Mathematics Subject Classification. Primary 42B25; Secondary 58C05, 60H99.

Key words and phrases. Fourier analysis, Gaussian measure, maximal function.

The fourth author was partially supported by CONICIT grant \#6970068. 
In order to prove Theorem [1] we need the following technical lemma, proved later.

Lemma 1. Let $B$ be a closed ball in $\mathcal{R}^{n}$ of radius $r$. Denote by $q$ the point of $B$ whose distance to the origin is minimal. Assume that $|q| \geq 1$ and that $r \geq 1 /|q|$. Then for all $x, y \in B$

$$
\gamma(B) \geq C \frac{e^{-|q|^{2}}}{|q|}\left(1 \wedge \frac{|y-x|^{2}}{|q|(|x| \vee|y|-|q|)}\right)^{\frac{n-1}{2}}
$$

Here and in the sequel, we write $C$ for various positive finite constants and denote $a \wedge b=\min (a, b)$ and $a \vee b=\max (a, b)$.

Proof of Theorem 1. We assume that $n \geq 2$, since the case $n=1$ is well known; see, e.g. 2]. Take $0 \leq f \in L^{p}(d \gamma)$ and $x \in \mathcal{R}^{n}$. For any ball $B$ containing $x$, we must estimate the average $\mathcal{A} f(B)=\frac{1}{\gamma(B)} \int_{B} f d \gamma$. Let $r$ and $q$ be defined as in Lemma 1 .

We first consider small balls $B$, and denote by $\mathcal{M}_{0} f(x)$ the supremum of $\mathcal{A} f(B)$ taken only over balls $B$ containing $x$ and verifying $r<1 \wedge|q|^{-1}$. Split $\mathcal{R}^{n}$ into rings $R_{k}=\{x: \sqrt{k-1} \leq|x|<\sqrt{k}\}, k=1,2, \ldots$. The width of $R_{k}$ is no larger than $1 / \sqrt{k}$, and so the Gaussian density is of constant order of magnitude in each $R_{k}$. Using Lebesgue measure arguments, one can easily estimate the $L^{p}(d \gamma)$ norm of $\mathcal{M}_{0} f$ in $R_{k}$ in terms of the $L^{p}(d \gamma)$ norm of $f$ in $\bigcup\left\{R_{k^{\prime}}:\left|k^{\prime}-k\right| \leq C\right\}$. This takes care of small balls.

Consider now balls $B$ with $r \geq 1 \wedge|q|^{-1}$. To begin with observe that the case $|q|<2$ is simple, since then $\gamma(B) \geq C$ and thus

$$
\mathcal{A} f(B) \leq C \int f d \gamma \leq C\|f\|_{L^{p}(d \gamma)}
$$

The corresponding part of $\mathcal{M} f$ thus satisfies the $L^{p}(d \gamma)$ estimate.

It remains to consider $\tilde{\mathcal{M}} f(x)=\sup \mathcal{A} f(B)$, the supremum taken over balls $B$ containing $x$ and with the property that $r \geq|q|^{-1}$ and $|q| \geq 2$. Let $B$ be such a ball, and observe that it satisfies the hypotheses of Lemma 1

For each $\rho \geq 1$ such that $S_{\rho}^{n-1}$ intersects $B$, let $y_{\rho} \in S_{\rho}^{n-1} \cap \partial B$ be such that $\left|y_{\rho}-x\right|=\sup _{z \in B \cap S_{\rho}^{n-1}}|z-x|$. Write $x^{\prime}=x /|x|$.

For each $z^{\prime} \in S^{n-1}$ such that $\rho z^{\prime} \in B$ we have

$$
\begin{aligned}
\left|x^{\prime}-z^{\prime}\right| & =\frac{1}{\rho}\left|\rho x^{\prime}-\rho z^{\prime}\right| \\
& \leq \frac{1}{\rho}\left[\left|x-\rho z^{\prime}\right|+|\rho-| x||\right] \\
& \leq \frac{2}{\rho}\left|y_{\rho}-x\right|
\end{aligned}
$$

and trivially $\left|x^{\prime}-z^{\prime}\right| \leq 2$. 
Because of (2) and the definition of $\mathcal{M}^{e}$,

(3)

$$
\begin{aligned}
\mathcal{A} f(B)= & \int_{|q|}^{|q|+2 r} \frac{1}{\gamma(B)} \int_{S^{n-1}} \chi_{B}\left(\rho z^{\prime}\right) f\left(\rho z^{\prime}\right) d \sigma\left(z^{\prime}\right) \rho^{n-1} e^{-\rho^{2}} d \rho \\
\leq & \int_{|q|}^{|q|+2 r} \frac{1}{\gamma(B)} \int_{\left|z^{\prime}-x^{\prime}\right| \leq 2\left(1 \wedge \frac{|y \rho-x|}{\rho}\right)} f\left(\rho z^{\prime}\right) d \sigma\left(z^{\prime}\right) \rho^{n-1} e^{-\rho^{2}} d \rho \\
\leq & C \int_{|q|}^{|q|+2 r} \frac{\left\{1 \wedge\left(\frac{\left|y_{\rho}-x\right|}{\rho}\right)^{n-1}\right\}}{\gamma(B)} \mathcal{M}^{e} f\left(\rho x^{\prime}\right) \rho^{n-1} e^{-\rho^{2}} d \rho \\
\leq & C \int_{|q|}^{|q|+2 r}|q| e^{|q|^{2}}\left\{1 \vee\left(\frac{|q|(\rho \vee|x|-|q|)}{\left|x-y_{\rho}\right|^{2}}\right)^{\frac{n-1}{2}}\right\}\left\{1 \wedge\left(\frac{\left|y_{\rho}-x\right|}{\rho}\right)^{n-1}\right\} \\
& \mathcal{M}^{e} f\left(\rho x^{\prime}\right) \rho^{n-1} e^{-\rho^{2}} d \rho,
\end{aligned}
$$

where we applied Lemma 1 with $y=y_{\rho}$ to get the last inequality.

Write $M=\rho \vee|x|$ and $m=\rho \wedge|x|$, so that $|q| \leq m \leq M$.

Lemma 2. For $|q|<\rho<|q|+2 r$ and some $C$,

$$
\begin{aligned}
& e^{|q|^{2}}\left\{1 \vee\left(\frac{|q|(M-|q|)}{\left|x-y_{\rho}\right|^{2}}\right)^{\frac{n-1}{2}}\right\}\left\{1 \wedge\left(\frac{\left|y_{\rho}-x\right|}{\rho}\right)^{n-1}\right\} \\
& \leq C e^{m^{2}}\left(\frac{1}{m^{2}} \vee \frac{M-m}{m}\right)^{\frac{n-1}{2}} .
\end{aligned}
$$

Assuming this lemma for the moment, we conclude from (3) that

$$
\mathcal{A} f(B) \leq C \int_{1}^{\infty} m e^{m^{2}}\left(\frac{1}{m^{2}} \vee \frac{M-m}{m}\right)^{\frac{n-1}{2}} \mathcal{M}^{e} f\left(\rho x^{\prime}\right) \rho^{n-1} e^{-\rho^{2}} d \rho .
$$

We split this integral into five integrals taken over the following intervals:

$$
\begin{aligned}
& I_{1}=\left[1, \frac{|x|}{2}\right], \quad I_{2}=\left(\frac{|x|}{2},|x|-\frac{1}{|x|}\right], \quad I_{3}=\left(|x|-\frac{1}{|x|},|x|+\frac{1}{|x|}\right], \\
& I_{4}=\left(|x|+\frac{1}{|x|}, \frac{5}{4}|x|\right], \quad I_{5}=\left(\frac{5}{4}|x|,+\infty\right) .
\end{aligned}
$$

Let for $i=1, \ldots, 5$

$$
\mathcal{M}_{i} f(x)=\int_{I_{i}} m e^{m^{2}}\left(\frac{1}{m^{2}} \vee \frac{M-m}{m}\right)^{\frac{n-1}{2}} \mathcal{M}^{e} f\left(\rho x^{\prime}\right) \rho^{n-1} e^{-\rho^{2}} d \rho .
$$

Then $\tilde{\mathcal{M}} f \leq C \sum_{1}^{5} \mathcal{M}_{i} f$.

Bound for $\mathcal{M}_{1} f(x)$. One finds that

$$
\mathcal{M}_{1} f(x) \leq|x|^{n} \int_{1}^{\frac{|x|}{2}} \mathcal{M}^{e} f\left(\rho x^{\prime}\right) d \rho .
$$


Hölder's inequality and the $L^{p}(d \sigma)$ boundedness of $\mathcal{M}^{e}$ imply

$$
\begin{aligned}
& \left\|\mathcal{M}_{1} f\right\|_{L^{p}(d \gamma)}^{p} \leq \int_{1}^{+\infty} \int_{S^{n-1}}\left(s^{n} \int_{1}^{\frac{s}{2}} \mathcal{M}^{e} f\left(\rho x^{\prime}\right) d \rho\right)^{p} d \sigma\left(x^{\prime}\right) s^{n-1} e^{-s^{2}} d s \\
& \leq \int_{1}^{+\infty} \int_{S^{n-1}} s^{n p} \int_{1}^{\frac{s}{2}}\left|\mathcal{M}^{e} f\left(\rho x^{\prime}\right)\right|^{p} \rho^{n-1} e^{-\rho^{2}} d \rho\left(\int_{1}^{\frac{s}{2}} \rho^{-(n-1) \frac{p^{\prime}}{p}} e^{\frac{p^{\prime}}{p} \rho^{2}} d \rho\right)^{\frac{p}{p^{\prime}}} d \sigma\left(x^{\prime}\right) s^{n-1} e^{-s^{2}} d s \\
& \leq\left(\int_{1}^{+\infty} s^{C} e^{-\frac{3}{4} s^{2}} d s\right)\|f\|_{L^{p}(d \gamma)}^{p} \leq C\|f\|_{L^{p}(d \gamma)}^{p} .
\end{aligned}
$$

Bound for $\mathcal{M}_{2} f(x)$. Making the change of variable $\rho=|x|-\frac{t}{|x|}$, we get

$$
\begin{aligned}
\mathcal{M}_{2} f(x) & \leq|x|^{\frac{n+1}{2}} \int_{|x| / 2}^{|x|-\frac{1}{|x|}}(|x|-\rho)^{\frac{n-1}{2}} \mathcal{M}^{e} f\left(\rho x^{\prime}\right) d \rho \\
& \leq \int_{1}^{|x|^{2} / 2} t^{\frac{n-1}{2}} \mathcal{M}^{e} f\left(\left(|x|-\frac{t}{|x|}\right) x^{\prime}\right) d t
\end{aligned}
$$

From Minkowski's integral inequality and the $L^{p}(d \sigma)$ boundedness of $\mathcal{M}^{e}$, we obtain

$$
\begin{aligned}
\left\|\mathcal{M}_{2} f\right\|_{L^{p}(d \gamma)} & \leq \int_{1}^{+\infty} t^{\frac{n-1}{2}}\left\|\mathcal{M}^{e} f\left(\left(|x|-\frac{t}{|x|}\right) x^{\prime}\right) \chi_{\left\{1 \leq t \leq \frac{|x|^{2}}{2}\right\}}\right\|_{L^{p}(d \gamma)} d t \\
& =\int_{1}^{+\infty} t^{\frac{n-1}{2}}\left[\int_{S^{n-1}} \int_{\sqrt{2 t}}^{+\infty} f\left(\left(s-\frac{t}{s}\right) x^{\prime}\right)^{p} s^{n-1} e^{-s^{2}} d s d \sigma\left(x^{\prime}\right)\right]^{\frac{1}{p}} d t .
\end{aligned}
$$

We now make the change of variables $s \rightarrow \rho=s-t / s$, observing that $s \leq 2 \rho$ and $-s^{2}=-\rho^{2}-2 t+t^{2} / s^{2} \leq-\rho^{2}-3 t / 2$ and $d \rho / d s \geq 1$. Thus

$$
\begin{aligned}
\left\|\mathcal{M}_{2} f\right\|_{L^{p}(d \gamma)} & \leq C \int_{1}^{+\infty} t^{\frac{n-1}{2}}\left[\left.\int_{S^{n-1}} \int_{\sqrt{t / 2}}^{+\infty}\left|f\left(\rho x^{\prime}\right)\right|\right|^{p} \rho^{n-1} e^{-\rho^{2}} e^{-3 t / 2} d \rho d \sigma\left(x^{\prime}\right)\right]^{\frac{1}{p}} d t \\
& \leq C\|f\|_{L^{p}(d \gamma)}\left(\int_{1}^{+\infty} t^{\frac{n-1}{2}} e^{-\frac{3 t}{2 p}} d t\right) \leq C\|f\|_{L^{p}(d \gamma)} .
\end{aligned}
$$

Bound for $\mathcal{M}_{3} f(x)$. Let $d \mu=\rho^{n-1} e^{-\rho^{2}} d \rho$ in $\mathcal{R}_{+}$. We have

$$
\begin{aligned}
\mathcal{M}_{3} f(x) & \leq C|x| \int_{|x|-1 /|x|}^{|x|+1 /|x|} \mathcal{M}^{e} f\left(\rho x^{\prime}\right) d \rho \\
& \leq C(\mu(|x|-1 /|x|,|x|+1 /|x|))^{-1} \int_{|x|-1 /|x|}^{|x|+1 /|x|} \mathcal{M}^{e} f\left(\rho x^{\prime}\right) d \mu(\rho) .
\end{aligned}
$$

Let $\mathcal{M}^{\mu}$ denote the one-dimensional centered maximal operator defined in terms of $\mu$, acting in the $\rho$ variable. Then

$$
\mathcal{M}_{3} f(x) \leq C \mathcal{M}^{\mu} \mathcal{M}^{e} f\left(|x| x^{\prime}\right) .
$$


But $\mathcal{M}^{\mu}$ is known to be bounded on $L^{p}(d \mu)$; see [1] or [2]. The $L^{p}(d \gamma)$ boundedness of $\mathcal{M}_{3}$ follows.

Bound for $\mathcal{M}_{4} f(x)$. Making the change of variable $\rho=|x|+\frac{t}{|x|}$, we have

$$
\begin{aligned}
\mathcal{M}_{4} f(x) & \leq C|x|^{\frac{n+1}{2}} e^{|x|^{2}} \int_{|x|+\frac{1}{|x|}}^{\frac{5}{4}|x|}(\rho-|x|)^{\frac{n-1}{2}} \mathcal{M}^{e} f\left(\rho x^{\prime}\right) e^{-\rho^{2}} d \rho \\
& \leq C \int_{1}^{\frac{|x|^{2}}{4}} t^{\frac{n-1}{2}} \mathcal{M}^{e} f\left(\left(|x|+\frac{t}{|x|}\right) x^{\prime}\right) e^{-2 t} e^{-\frac{t^{2}}{|x|^{2}}} d t .
\end{aligned}
$$

Minkowski's integral inequality implies

$$
\left\|\mathcal{M}_{4} f\right\|_{L^{p}(d \gamma)} \leq C \int_{1}^{+\infty} t^{\frac{n-1}{2}}\left\|\mathcal{M}^{e} f\left(\left(|x|+\frac{t}{|x|}\right) x^{\prime}\right) e^{-\frac{t^{2}}{|x|^{2}}} \chi_{\left\{1 \leq t \leq \frac{|x|^{2}}{4}\right\}}\right\|_{L^{p}(d \gamma)} e^{-2 t} d t
$$

But $\mathcal{M}^{e}$ is bounded on $L^{p}(d \sigma)$, so that

$$
\begin{gathered}
\left\|\mathcal{M}^{e} f\left(\left(|x|+\frac{t}{|x|}\right) x^{\prime}\right) e^{-\frac{t^{2}}{|x|^{2}}} \chi_{\left\{1 \leq t \leq \frac{|x|^{2}}{4}\right\}}\right\|_{L^{p}(d \gamma)}^{p} \\
\leq C \int_{2 \sqrt{t}}^{\infty} \int_{S^{n-1}}\left|f\left(\left(s+\frac{t}{s}\right) x^{\prime}\right) e^{-\frac{t^{2}}{s^{2}}}\right|^{p} d \sigma\left(x^{\prime}\right) s^{n-1} e^{-s^{2}} d s .
\end{gathered}
$$

Almost as in the case of $\mathcal{M}_{2}$, we make the change of variable $\rho=s+t / s$ and observe that $s \leq \rho$ and $-s^{2}=-\rho^{2}+2 t+t^{2} / s^{2}$ and $d \rho / d s \geq 1 / 2$. Since $e^{-p t^{2} / s^{2}} e^{t^{2} / s^{2}}<1$, it follows that the above double integral is at most

$$
C \int_{S^{n-1}} \int_{1}^{+\infty}\left|f\left(\rho x^{\prime}\right)\right|^{p} \rho^{n-1} e^{-\rho^{2}} d \rho d \sigma\left(x^{\prime}\right) e^{2 t} \leq C\|f\|_{L^{p}(d \gamma)}^{p} e^{2 t} .
$$

Thus

$$
\left\|\mathcal{M}_{4} f\right\|_{L^{p}(d \gamma)} \leq C \int_{1}^{+\infty} t^{\frac{n-1}{2}}\|f\|_{L^{p}(d \gamma)} e^{\frac{2 t}{p}} e^{-2 t} d t \leq C\|f\|_{L^{p}(d \gamma)} .
$$

Bound for $\mathcal{M}_{5} f(x)$. Observe that

$$
\mathcal{M}_{5} f(x) \leq|x|^{\frac{3-n}{2}} e^{|x|^{2}} \int_{\frac{5}{4}|x|}^{+\infty} \mathcal{M}^{e} f\left(\rho x^{\prime}\right) \rho^{\frac{n-1}{2}} \rho^{n-1} e^{-\rho^{2}} d \rho .
$$

We take the $L^{p}$ norm and then apply Hölder's inequality, getting

$$
\begin{aligned}
&\left\|\mathcal{M}_{5} f\right\|_{L^{p}(d \gamma)}^{p} \leq \int_{1}^{+\infty} \int_{S^{n-1}} \frac{e^{p s^{2}}}{s^{p \frac{n-3}{2}}}\left(\int_{\frac{5 s}{4}}^{+\infty} \mathcal{M}^{e} f\left(\rho x^{\prime}\right) \rho^{\frac{3(n-1)}{2}} e^{-\rho^{2}} d \rho\right)^{p} d \sigma\left(x^{\prime}\right) s^{n-1} e^{-s^{2}} d s \\
& \leq \int_{1}^{+\infty} \int_{S^{n-1}} \frac{e^{p s^{2}}}{s^{p \frac{n-3}{2}}} \int_{0}^{+\infty}\left|\mathcal{M}^{e} f\left(\rho x^{\prime}\right)\right|^{p} \rho^{n-1} e^{-\rho^{2}} d \rho\left(\int_{\frac{5 s}{4}}^{+\infty} \rho^{\left(\frac{p^{\prime}}{2}+1\right)(n-1)} e^{-\rho^{2}} d \rho\right) d \sigma\left(x^{\prime}\right) s^{n-1} e^{-s^{2}} d s \\
& \leq\|f\|_{L^{p}(d \gamma)}^{p}\left(\int_{1}^{+\infty} s^{C} e^{(p-1) s^{2}} e^{-(p-1)\left(\frac{5}{4} s\right)^{2}} d s\right) \\
& \leq C\|f\|_{L^{p}(d \gamma)}^{p} \cdot
\end{aligned}
$$

To finish the proof of Theorem 1 , it now only remains to prove the two lemmas. 
Proof of Lemma 1. Consider the hyperplane orthogonal to $q$ whose distance from the origin is $|q|+t$, with $1 /(2|q|)<t<1 /|q|$. Its intersection with $B$ is an (n1)-dimensional ball whose radius is at least $C \sqrt{r t} \geq C \sqrt{r /|q|}$. Integrating the Gaussian density first along this $(n-1)$-dimensional ball and then in $t$, we get

$$
\gamma(B) \geq \int_{1 /(2|q|)}^{1 /|q|} e^{-(|q|+t)^{2}} d t \int_{|v|<C \sqrt{r /|q|}} e^{-|v|^{2}} d v,
$$

where $v$ is an $(n-1)$-dimensional variable. The inner integral here is at least $C \min \left(1,(r /|q|)^{(n-1) / 2}\right)$, and $e^{-(|q|+t)^{2}} \geq C e^{-|q|^{2}}$ for these $t$; therefore

$$
\gamma(B) \geq C \frac{e^{-|q|^{2}}}{|q|}\left(1 \wedge\left(\frac{r}{|q|}\right)^{\frac{n-1}{2}}\right) .
$$

To estimate $r$ from below, we let $z$ be the center of $B$ and $w$ the projection of $x$ onto the line passing through $0, q$ and $z$. Write $h=|x-w|$ and $a=|w-q|$. Applying the Pythagorean Theorem twice, we get

$$
|x-z|^{2}-(r-a)^{2}=h^{2}=|x-q|^{2}-a^{2} .
$$

Since $|x-z| \leq r$, we conclude that $2 a r \geq|x-q|^{2}$. Clearly $a \leq|x|-|q|$ so that

$$
r \geq \frac{|x-q|^{2}}{2(|x|-|q|)} \geq \frac{|x-q|^{2}}{2(|x| \vee|y|-|q|)}
$$

Since $x$ and $y$ are arbitrary points of $B$, the same argument also implies

$$
r \geq \frac{|y-q|^{2}}{2(|x| \vee|y|-|q|)} .
$$

From the triangle inequality we conclude that $2|x-q| \vee|y-q| \geq|x-y|$, and so

$$
r \geq \frac{|x-y|^{2}}{8(|x| \vee|y|-|q|)} .
$$

Combining this with (4), we obtain the inequality of Lemma 1 .

Proof of Lemma 国. We write LHS for the left-hand side of the inequality to be proved. Assume first that

$$
\left(\frac{|q|(M-|q|)}{\left|x-y_{\rho}\right|^{2}}\right)^{\frac{n-1}{2}} \leq 1
$$

Then LHS $\leq e^{|q|^{2}}\left(\left|x-y_{\rho}\right| / \rho\right)^{n-1}$. The angles at $q$ of the triangles $0 q x$ and $0 q y_{\rho}$ are obtuse, so that $|x|^{2} \geq|q|^{2}+|x-q|^{2}$ and $\left|y_{\rho}\right|^{2} \geq|q|^{2}+\left|y_{\rho}-q\right|^{2}$. But $\left|x-y_{\rho}\right| \leq$ $|x-q|+\left|y_{\rho}-q\right|$, and this implies

$$
\begin{aligned}
\left|x-y_{\rho}\right|^{2} & \leq 4 \max \left(|x-q|^{2},\left|y_{\rho}-q\right|^{2}\right) \\
& \leq 4 \max \left(|x|^{2}-|q|^{2},\left|y_{\rho}\right|^{2}-|q|^{2}\right)=4\left(M^{2}-|q|^{2}\right) .
\end{aligned}
$$

If $|x| \leq 2 \rho$, this last quantity is at most $16 \rho(M-|q|)$, and then

$$
\text { LHS } \leq C e^{|q|^{2}}\left(\frac{M-|q|}{\rho}\right)^{\frac{n-1}{2}} .
$$

In the contrary case $|x|>2 \rho$, we simply observe that LHS $\leq C e^{|q|^{2}}$ whereas the right-hand side is at least $C e^{m^{2}}$. This case of the lemma is thus trivial. 
Assume now that (5) is false. Then

$$
\text { LHS } \leq e^{|q|^{2}} \frac{(|q|(M-|q|))^{\frac{n-1}{2}}}{\rho^{n-1}}
$$

and we arrive again at (6).

It thus only remains to see that (6) implies Lemma 2, This would follow from the estimate

$$
e^{|q|^{2}-m^{2}}(M-|q|)^{\frac{n-1}{2}} \leq C((1 / m) \vee(M-m))^{\frac{n-1}{2}} .
$$

To prove (7), we use the fact that

$$
(M-|q|)^{\frac{n-1}{2}} \leq C\left((M-m)^{\frac{n-1}{2}}+(m-|q|)^{\frac{n-1}{2}}\right)
$$

and when $m-|q|>1 / m$ also

$$
e^{|q|^{2}-m^{2}}=e^{-(m-|q|)(m+|q|)} \leq \frac{C}{(m-|q|)^{\frac{n-1}{2}} m^{\frac{n-1}{2}}} .
$$

Now (77) and Lemma 2 follow.

\section{REFERENCES}

[1] Muckenhoupt, B., Poisson integrals for Hermite and Laguerre expansions. Trans. Amer. Math. Soc. 139 (1969), 231-242. MR 40:3158

[2] Sjögren, P., A remark on the maximal function for measures in $R^{n}$. Amer. J. Math. 105 (1983), 1231-1233. MR 86a:28003

[3] Sjögren, P. and Soria, F., Sharp estimates for the noncentered maximal operator associated to Gaussian and other radial measures. Preprint.

[4] Vargas, A.M., On the maximal function for rotation invariant measures in $R^{n}$. Studia Math. 110 (1) (1994), 9-17. MR 95e:42019

Department of Mathematics, Universidad Nacional del Litoral and CONiCET, ArGENTINA

E-mail address: forzani@pemas.unl.edu.ar

Department of Mathematics, Universidad Nacional de Salta, Argentina

E-mail address: scotto@math.unl.edu.ar

Department of Mathematics, Göteborg University, SE-412 96 Göteborg, Sweden

E-mail address: peters@math.chalmers.se

School of Mathematics, Universidad Central de Venezuela, Caracas 1040, Venezuela

E-mail address: wurbina@euler.ciens.ucv.ve 